\title{
Complementos a la Laparoscopia Diagnóstica Ginecobstétrica
}

\author{
Conferencia presentada a nombre de \\ la SOCIEDAD COLOMBIANA DE ME- \\ DICOS ENDOSCOPISTAS, ante el XIV \\ CONGRESO NACIONAL DE GINE- \\ COBSTETRICIA reunido en la ciudad de \\ Cartagena en diciembre de 1981, auspi- \\ ciada por PROFAMILIA, y elaborada \\ por el Doctor GUSTAVO CASAS VAS- \\ QUEZ.
}

Medell ín

En el transcurso de la exploración ginecológica por medio de laparoscopia, que Kurt Semm denominara pelviscopia, nos encontramos los ginecólogos laparoscopistas necesariamente con una serie de hechos diferentes a los que nos son ya familiares durante el diágnostico diferencial ginecológico y obstétrico en la laparoscopia pélvica.

Estos hechos normales y anormales surgen de la inevitable necesidad de mirar la cavidad abdominal como un todo, en el trascurso de este proceso diagnóstico.

La inquietud que nos produjo en un principio encontrarnos con hallazgos que no sabíamos interpretar bien (la cual seguramente ustedes comparten con nosotros), nos ha llevado a plantear en esta reunión las bases para la interpretación correcta de la signología laparoscópica diferente a la ginecobstétrica que conduzca a brindarle un diagnóstico adecuado y una terapéutica eficaz al paciente que se nos ha encomendado.
No pretendemos convertirnos en internistas o gastroenterólogos que definan con precisión mediante la laparoscopia el diagnóstico a que se llega mediante este tipo de visión endoscópica, pero si creemos que es indispensable para el ginecobstetra laparoscopista definir la presencia o ausencia de patología a nivel de los órganos abdominales, e intentar darle un nombre específico.

Es bien sabido que la laparoscopia pélvica se ha difundido en Colombia y en el mundo con una extraordinaria rapidez; en cambio, la subespecialidad de laparoscopistas de los órganos abdominales superiores carece de suficiente número de colegas que la practiquen en nuestro medio.

Es así como desde hace varios años se nos está remitiendo continuamente a Profamilia Medellín pacientes de ambos sexos provenientes de diferentes entidades de salud, en los cuales se requiere esclarecer la presencia de enfermedades no relacionadas con la ginecología. 
Fuera de esto tenemos que admitir que incidentencialmente durante el desempeño de una tubectomía laparoscópica, o durante el desarrollo de un diagnóstico ginecobstétrico, nos encontramos con imágenes laparoscópicas cuya entidad es un deber para nosotros definir.

\section{Metodología}

En primer lugar mencionemos que para lograr una adecuada evaluación del hemiabdómen superior es necesario contar con una mesa laparoscópica de carácter diferente a la obstétrica, la cual nos permite desplazar al paciente hacia las posiciones adecuadas para la visualización de los órganos que van a estudiarse; por lo tanto la mesa debe ser quirúrgica $y$ de excelentes cualidades.

En segundo lugar vemos que la anestesia local parece ser la preferida de quienes tienen experiencia en el diagnóstico laparoscópico propiamente dicho.

En tercer lugar, todo paciente que vaya a someterse a un estudio laparoscópico de esta índole debe traer una historia clínica muy bien elaborada, complementada con exámenes de laboratorio que hayan creado ya un diagnóstico presuntivo que nos permita saber si no hay contraindicaciones absolutas para practicarle el examen en sí, o biopsias del hígado, por ejemplo. Entre estos exámenes se destaca la necesidad del recuento de plaquetas y el tiempo de protrombina.

\section{Técnica y complicaciones}

La paciente debe permanecer en ayunas desde la noche anterior, se recomienda la provisión de un enema evacuante la noche víspera.

PREMEDICACION. De acuerdo al tipo específico de paciente y según la historia clínica será necesario asesorar- nos del consejo del anestesiólogo para proveer la promedicación más adecuada a cada caso en particular.

ANESTESIA. Se prefiere la anestesia local como la Lidocaína, acompañada de una sedacción la cual aconsejará también el anestesiólogo que nos asesora.

NEUMOPERITONEO. Con la aguja de Verres, y eligiendo un punto situado a dos traveses de dedo por encima del ombligo y un través de dedo a su izquierda, practicamos el neumoperitoneo y por este mismo sitio la introducción del trocar. Para estas maniobras se tendrán las mismas precauciones que para la pelviscopia.

Sin embargo como es lo usual, la detección de la patología abdominal deberá ocurrir en el transcurso de la práctica de una laparoscopia de investigación ginecobstétrica, lo cual simplemente nos aleja un tanto de los órganos superiores que deseamos evaluar.

\section{Organos asequibles al diagnóstico laparoscópico}

El estómago, el duodeno, el páncreas, el intestino delgado, y la mayor parte del colon, tienen sus mejores métodos exploratorios en los Rayos X, en la endos. copia directa intraluminal y en otros métodos de laboratorio.

Hay algunos sectores del intestino grueso que se pueden explorar por medio de laparoscopia, por lo menos en sus aspectos externos, como son el apéndice cecal $y$ el ciego, el sigmoides y el recto

Los órganos que mayor intormación proveen al laparoscopista son el hígado, la vesícula biliar, el omento, el ápendice cecal, el peritoneo parietal y visceral, porciones del estómago y el bazo.

Las posiciones adecuadas para lograr la mejor visualización de los órganos 
abdominales se denominan posiciones uno, dos, tres $\mathrm{y}$ cuatro de Henning $\mathrm{y}$ Look.

\section{Exploraciones complementarias}

De todas las que pueden realizarse en el curso de la laparoscopia merecen especial mención en este papel la biopsia hepática dirigida, puesto que la esplenoportografía, la colescistocolangiografía y otras, son del dominio y exclusiva competencia de los subespecialistas.

\section{La biopsia hepática}

Se ha practicado desde hace ya tiem po con mucho entusiasmo por los inter. nistas y cirujanos generales. Sin embargo el hecho de caer o no sobre el área afectada del hígado con la aguja de biopsia, crea la gran diferencia que tiene a su favor la biopsia dirigida por laparoscopia.

La aguja de punción debe ingresar al órgano en un ángulo de 20 a 40 grados de inclinación respecto al plano de la superficie hepática y de preferencia so bre el lóbulo derecho a fin de evitar accidentes de punción, a vasos grandes y conductos biliares. El área perivesicular está absolutamente vedada para la toma de biopsias por razones obvias.

Revisemos rápidamente cuales son las contraindicaciones absolutas para la práctica de la biopsia dirigida:

Trastornos de la coagulación (tiempo de Protrombina de $30 \%$ o menos, recuento de plaquetas de $50 \mathrm{mil}$ o menos) hemofilia, ictericia obstructiva extra hepática, colangitis aguda, colestasis, hígado hemangiomatoso y quístico.

Las complicaciones de la biopsia hepática son tres: la hemorragia, la colerraqia y la punción de quistes hidatídicos.
HEMORRAGIA. La verificación de las contraindicaciones la evita, pero si ocurre un sangrado por un tiempo mayor de cinco a diez minutos se puede controlar mediante la comprensión del orificio sangrante, o la cauterización, o la aplica. ción de la adrenalina local al $1 \%$ o la aplicación de 100 unidades de trombina.

La COLERRAGIA. Es difícil de con. trolar por lo tanto se debe evitar la punción de todo hígado que tenga coloración verdosa; en caso de presentarse se recomienda inyectar sangre del mismo paciente en el conducto biliar roto seguido de un hemostático que provoque su coagulación; pero la medida más eficaz parece ser la colocación de un tubo de drenaje en las proximidades del orificio de la punción.

En vista de la experiencia que hemos tenido en Medellín, con los accidentes colerrágicos, aún en caso de hígados no colestáticos, nos permitimos recomendar la cauterización rutinaria de toda biopsia hepática aunque el sangrado sea mínimo.

En el raro caso de encontrarnos un quiste hidatídico que hemos puncionado accidentalmente, la mejor medida sería aspirar todo el contenido, formolizarlos y practicar enseguida su resección quirúrgica inmediata.

Las contraindicaciones absolutas de la laparoscopia digestiva y hepática son las mismas de la ginecológica

\section{DETECCION DE ANORMALIDADES A NIVEL DEL PERITONEO INTESTINO, ESTOMAGO, EPIPLON Y LIGAMENTOS}

La pared abdominal es un buen lugar para registrar cambios a nivel del peritoneo. A este nivel la serosa se sitúa bajo la fascia aponeurótica en su porción inferior $y$ bajo las inserciones diafragmáticas en la porción superior. 
Por ello presenta una coloración blanca y grisácea en la zona que recubre la pared abdominal anterior y roja en la porción más alta del abdómen. Toda su superficie aparece lisa y brillante, distendidas las paredes abdominales por el neumoperitoneo, con un foco de refle: xión luminosa bien definido.

Del ombligo hacia abajo se observan los ligamentos umbilicales medio y lateral. Cuando el paciente es obeso el peritoneo se colora de amarillo y pueden pender de él, ápendices adiposos. Aunque el peritoneo es muy rico en vasos, laparoscópicamente son poco llamativos.

\section{Alteraciones peritoneales}

Aumento del fluido peritoneal: normalmente el peritoneo brilla por la presencia de un pequeño trasudado. Cuando aumenta se acumula entre las visceras, en el Douglas y en los flancos. Cuando la ascitis es masiva todos los órganos quedan recubiertos por ella. El intestino y el epilplón quedan flotando moviéndose al ritmo del pulso aórtico y de la respiración.

\section{Trasudado}

Es amarillento claro y transparente, coexiste frecuentemente con signos de hipertensión portal y con patología hepática, especialmente con el hígado card ıaco. Cuando el trasudado es antiguo se torno opaco y blanquecino recordando la ascitis quilosa.

EL EXUDADO. Es menos fluido opalescente con grumos de fibrina. Pero la sola observación de estas características no es suficiente para diferenciarlo del trasudado pues ya hemos visto que cuando este es antiguo se opacifica, se acompaña generalmente de signos de inflamación peritoneal, hiperemia y adherencias. La ascitis hemorrágica sugiere la presencia del tumor maligno. La ascitis quilosa tiene un color blanco lechoso, se explica por un bloqueo linfático a nivel de adenopatía abdominal o del conducto toráxico.

ALTERACIONES DEL ESPESAMIENTO PERITONEAL. Cuando este fenómeno ocurre, el peritoneo pierde su trasparencia; se torna opaco y turbio $y$ con reflejos luminosos deformados o descompuestos. La ascitis de cierta duración produce este cambio. El depósito de fibrina o la simple irritación mecánica son la causa de este engrosamiento. Estos cambios son generalizados y por lo tanto afectan también la vesícula, la cual cambia su coloración normal azulada a grisácea o blanquecina. Acompañan a los procesos inflamatorios, pero también se ve en la cirrosis:.

Las fibrosis pueden ser o no significativas según se acompañen de otros signos como las adherencias y recordatorios de algún trauma quirúrgico o similàr.

PERITONITIS. EI enrojecimiento, edema, vascularización aumentada, depósitos de fibrina, son las causas del engrosamiento peritoneal que acompañan la peritonitis. En algunas formas de tumores secundarios todo el peritoneo se encuentra engrosado en ocasiones de aspecto irregular blanquecino y en otras gelatinoso.

ALTERACIONES VASCULARES. EI aumento de la trama vascular ocurre por tres mecanismos: inflamación, hipertensión portal, y reflejo vaso-motor, este último se reconoce por su pronta desaparición o cambio de apariencia, y está producido por estímulos mecánicos o térmicos en el dermatoma correspondiente. Los signos inflamatorios pueden afectar zonas específicas, por ejemplo el peritoneo perihepático en casos de hepatitis, el perivesicular en casos de colecistitis, la fosa ilíaca derecha (V. 8) en caso de apendicitis. La hiperemia acom- 
paña a las peritonitis crónicas tuberculosas, a las carcinomatosis, y a las enfermedades periódicas.

HIPERTENSION PORTAL. Vasos muy ingurgitados de curso irregular, serpenteantes anastomosados en red de densidad y espesor variables. La estasis venosa se reconoce por la coloración más oscura, a veces cianótica. En los casos iniciales estos cambios se descubren sólo en los flancos y en el ligamento frenocólico

NODULOS PERITONEALES. Pueden ser de dos tipos: inflamatorios y tumorales. Los inflamatorios, como en la tuberculosis, son generalmente homogéneos y rodeados de hiperemia, con exudado fibrinoso y/o adherencias, sólidos y blanquecinos aplanados. Los nódulos tumorales en cambio son generalmente heterogéneos con escasa hiperemia $y$ exudado generalmente ausente, si son redondeados o sésiles, su confusión es más difícil. De todas maneras deben diferenciarse mediante la biopsia. Algunas metástasis iniciales adoptan figura lenticular. La esteatonecrosis adopta también un aspecto macular, aparece como espiculaciones calcáreas de bordes irregulares.

ADHERENCIAS. Respecto a las adhe rencias tenemos tantos lugares comunes que no es necesario entrar en amplia explicación para interpretar su significado, pero debemos recordar que si éstas aparecen a nivel del hígado, podemos suponer la existencia de hidatidosis, abscesos subfrénicos, colangitis supurativas, absceso amebiano y hepatopatías inflamatorias.

\section{ENFERMEDADES DEL ESTOMAGO $Y$ EL INTESTINO}

ESTOMAGO. Consideran autoridades en la materia que la definición de la patología gástrica por medio de la laparoscopia es aventurada y, por lo tanto, no me detengo a analizarla.

INTESTINO DELGADO. La patología reflejada a nivel del peritoneo visceral del intestino, será la que mejor podamos analizar dentro de el intento de una definición laparoscópica. El reconocimeinto de la patología a nivel del intestino grueso lo haremos basándonos en la presencia de cintillas longitudinales estriadas que lo recorren.

El ápendice cecal normal se nos ofrece con su' aspecto vermicular con vasos finos subserosos. La práctica sistemática de la laparoscopia en dolores abdominales agudos de naturaleza dudosa nos permite identificar la apendicitis aguda: el apéndice aparece aumentado de volúmen $y$ enrojecido, con aspecto de porra o salchicha, a veces con sufuciones hemorrágicas. Cuando se inicia la gangrena esta se reconoce por la presencia de áreas más grisáceas o azuladas, con abundamate exudado fibrinoso. Cuando se ha perforado, puede encontrarse un acumulo purulento. En las apendicitis retrocecales, quizas el único signo diagnóstico sea la presencia de peritoeno hiperémico en la vecindad.

Otras afecciones inflamatorias de la vecindad pueden crear un cuadro visual igual y por lo tanto conducir a un diagnóstico errado.

EPIPLON MAYOR. Sus características normales nos son a los ginecobstetras bien conocidas, pero vale la pena recordar que cubre con frecuencia órganos que deseamos visualizar y que su desplazamiento activo o pasivo nos permite lograr nuestro objetivo. El omento es asiento de una rica patología ya descrita y mencionada; sin embargo recordemos aquí la presencia de los vasos lumbricoides azulados en casos de hipertensión portal. Su retracción en muñón cilíndrico es frecuentemente hallada en las 
infiltraciones carcinomatosas y algunas tuberculosas. La carcinomatosis peritoneal lo puede transformar en una lámina gruesa y rígida blanquecina ondulada y tumoral.

LIGAMENTO REDONDO. Une la línea media del ombligo con la hendedura interlobular, sus características normales son variables, desde delgada cintilla hasta adiposo cordón. Su desplazamiento en otras direcciones se observa con motivo de cambios del tamaño hepático (atrofias e hipertrofias).

LIGAMENTO FALCIFORME. Tras el ligamento redondo y generalmente adosado a él se ve una lámina azulada semitrasparente que divide la cavidad abdominal superior en dos mitades. Los cambios que a este nivel se aprecian estan relacionados con la hipertensión portal.

\section{VESICULA BILIAR}

Visible en la posición uno de Henning; mediante elevación de la cabeza y descenso de las extremidades inferiores se consigue su mejor visualización; en ocasiones no es visible debido a hepatomegalia, en cuyo caso es necesario elevar el borde hepático.

CARACTERISTICAS. - TAMAÑO. Normalmente se sitúa su fondo a nivel del borde hepático y a veces lo sobrepasa ligeramente.

VESICULAS GRANDES. Se habla de ellas cuando el fondo se sitúa a uno o varios centímetros por debajo del borde hepático. Puede tratarse de simples variaciones anatómicas pero también de hepatopatías. En las hepatitis la vesícula aparece hipotónica y dilatada manteniéndose así en las hepatitis crónicas. En las cirrosis $y$ en cualquier alteración profunda de la función hepática como las metástasis $p(V I .3)$. La dilatación en las hepatopatías comprometen la capacidad contráctil de todo el sistema biliar. Al contrario de lo que ocurre en las obstrucciones distales, donde hay dilatación marcada y tensión interna aumentada por ello, su fondo se halla levantado. Cuando la tensión interna no es tan alta, la bilis se acumula en el fondo y adopta una forma esférica pequeña. Para confirmar la alta tensión interna, simplemente observamos que al presionarla con algún instrumento la depresión se recupera de inmediato. En estos casos es frecuente encontrar un h ígado colestácico.

La obstrucción del cístico por cálculos causa una gran distensión. En estos casos aparecen signos de inflamación crónica y quizá se detecta con la palpación la presencia de litiasis.

Las inflamaciones agudas distienden la vesícula y las crónicas tienden a retraerla. Si la vesícula no se visualiza, podemos levantar el hígado con el laparoscopio y observar si se trata de un proceso postevacuador normal o de un estado patológico.

Cuando hay obstrucción en el hilio hepático, la vesícula aparece vacía y con escaso contenido en su interior. Igual fenómeno ocurre en la hepatopatía agu. da colestática.

En las colacistitis crónicas, la vesícula aparece retraida y escleroatrófica de muy difícil visualización.

FORMA. Es normal el aspecto fusiforme y en gorro frigio. La litiasis puede deformar la vesícula y al palparla comprobamos que en esa zona no se deprime; la inflamación crónica también deforma la vesícula.

En sus estadios iniciales el carcinoma de la vesícula no se logra diferenciar de la colecistitis crónica; cuando ya está avanzado, sus deformidades nodulares y tuberosas son fáciles de reconocer.

COLOR DE LA PARED. Sus paredes son delgadas de coloración azulosa, ver- 
dosa o gris oscura. Cuando la pared aumenta de espesor adquiere una tonalidad blanquecina o gris pálida. Uno de los signos de colecistitis crónica es este engrosamiento parietal. Cuando sobre la pared se deposita calcio, adquiere un color blanco porcelana, es dura y resistente a la comprensión. El enrojecimiento parietal se observa en la fase más aguda de la colecistitis. La vesícula no tiene normalmente adherencias, pero en una cuarta parte de las normales existe el denominado ligamento fetal, que remeda las adherencias pero no se acompaña de ningún signo inflamatorio agudo o crónico. En las adherencias de origen neoplásico, su prominencia sugie. re la naturaleza tumoral.

En resumen, las enfermedades de la vesícula para el ginecobstetra pueden resumirse así: 1, COLECISTITIS AGUDA: enrojecimiento difuso y homogéneo de la pared, aumento del tamaño y de su tensión interna, exudación fibrinosa en su alrededor.

El hallazgo de exudación purulenta y biliar representa signos de emergencia que requieren atención inmediata, 2, colecistitis crónica; la vesícula está con frecuencia oculta por adherencias, que son producto de sucesivos brotes agudos. Es costante el engrosamiento parietal y con frecuencia hay hiperemia.

3 , carcinoma vesicular suele ser indiferenciable de la colecistitis crónica. Puede presentar áreas de mayor engrosamiento o adherencias confluentes. En la infiltración tumoral del lecho hepático, esta zona toma una coloración blanquecina extendida como una mancha de aceite cuyo epicentro es la vesícula; posee vasos tortuosos con paredes blancas y más duras a la palpación.

\section{HIGADO}

\section{Semiología laparoscópica}

HEPATOMEGALIAS. Hay dos formas para definirlas: colocando nuestros dedos contra el reborde costal para ver si el borde hepático coincide o lo rebasa, o mejor observando la insersión del ligamento redondo, el cual normalmente no debe ser sobrepasado por ninguno de los dos lóbulos.

Los hígados pequeños son bien raros y generalmente representan el resultado de una hepatitis crónica agresiva necrotizante.

SUPERFICIE HEPATICA. Para su estudio se aconseja observar la forma como se refleja la luz de la lámpara. Si la superficie es lisa se reconocerá con facilidad la clara reflexión del foco luminoso. Si el reflejo luminoso está deformado podemos esperar alteraciones importantes del hígado. Si el reflejo luminoso se fragmenta en forma de gránulos $\mathrm{u}$ ondas aplanadas y amplias, el cuadro corresponde generalmente al de una hepatitis, mientras que si lo hace en nodulaciones puede corresponder a una cirrosis. Si al mismo tiempo observamos elementos de hipertensión portal tendremos requisitos fundamentales para hablar de cirrosis. Creemos que no es de nuestra competencia entrar a clasificar por medio de la laparoscopia los diferentes tipos de cirrosis.

BORDE HEPATICO. Aunque el borde y la superficie hepática lisos finos y blandos no sean totalmente específicos del hígado normal esto es lo que debe esperarse normalmente de él. Los bordes finos y fibrosos los bordes festoneados y a veces aserrados, son casi siempre signos de hígado anormal según se ha comprobado por medio de biopsias hepáticas múltiples. Su causa se puede enmarcar dentro de las hepatitis crónicas y las cirrosis. Generalmente se asocian con otra alteración estructural de la superficie.

Los bordes engrosados y romos generalmente corresponden a problemas inflamatorios y edematosos, a infiltración grasa, etc. 
COLORACION DEL HIGADO. Establecer un diagnóstico exclusivamente sobre la base de su coloración acarrea errores para la interpretación diagnóstica; sin embargo para el ginecobstetra laparoscopista es importante saber que el hígado verdoso implica siempre colestasis y es aquél en el cual la biopsia hepá tica está contraindicada.

NODULOS HEPATICOS. Si son blanquecinos generalmente son metastásicos y la mayor parte de las veces se acompañan de umbilicación central a diferencia de los nódulos de tipo linformatoso. Los nódulos rojos carnosos corresponden. generalmente a adenomas: igualmente se presentan en el hígado granos de diversa etiologîa la cual se definirá más fácilmente durante la biopsia.
Esta charla ha pretendido ser un abrebocas para despertar la inquietud por el estudio concienzudo de la patología correspondiente a los órganos abdominales no pélvicos.

\section{Bibliografía}

1. SOLIS-HERRUZO J.A. Atlas de Diagnóstico Diferencial Laparoscópico. Editorial Paz Montalvo. 1975.

2. SEEM. K. Atlas Of Gynecolog Laparoscopy and Hysteroscopy. W.B. Saunders Company. 1977.

3. CASAS Gustavo y COLS. EI Diagnóstico Diferencial en la Laparoscopia. Trabajo presentado al Congreso de Ginecología y Obstetricia reunida en la ciudad de Bogotá, en diciembre de 1979. 